\title{
A INFLUÊNCIA DO MODO DE OPERAÇÃO DO SSSC NA ESTABILIDADE DE ÂNGULO DE SISTEMAS ELÉTRICOS DE POTÊNCIA
}

\author{
Marcelo S. Castro* \\ mcastro@dsee.fee.unicamp.br \\ Igor Kopcak* \\ kopcak@dsee.fee.unicamp.br
}

\author{
Hugo M. Ayres* \\ hmayres@dsee.fee.unicamp.br \\ Vivaldo F. da Costa* \\ vivaldo@dsee.fee.unicamp.br
}

\author{
Luiz Carlos P. da Silva* \\ lui@dsee.fee.unicamp.br
}

* Departamento de Sistemas de Energia Elétrica, Universidade Estadual de Campinas

Caixa Postal 6101, 13083-852, Campinas, São Paulo, Brasil

\begin{abstract}
In order to accomplish specific compensation objectives, a Static Synchronous Series Compensator (SSSC) can be controlled according different methods. In the literature, the most discussed control modes of the SSSC are 1) constant voltage mode, 2) constant impedance emulation mode, and 3 ) constant power control mode. The first two modes above cited are used when purely series reactive compensation is required, whereas the third one also enables to control the power flow in the transmission network. Since these modes can have different impacts on system angle stability, this paper aims to assess the influence of SSSC operation mode on both small-signal and transient stability of power systems. The study is based on modal analysis and time domain simulations which are carried out by using the Power System Analysis Toolbox (PSAT). The results obtained allow to conclude that, when performing purely series reactive compensation, the SSSC usage in the constant impedance emulation mode is the most beneficial strategy to improve both smallsignal and transient stability.
\end{abstract}

KEYWORDS: FACTS, PSAT, small-signal stability, SSSC, transient stability.

\footnotetext{
Artigo submetido em 03/02/2006

1a. Revisão em 06/10/2006

2a. Revisão em 24/04/2007

Aceito sob recomendação do Editor Associado Prof. Carlos A. Castro
}

\section{RESUMO}

Afim de realizar específicos objetivos de compensação, um SSSC (Static Synchronous Series Compensator) pode ser controlado de diferentes formas. Os modos de controle do SSSC mais discutidos na literatura são 1) modo de tensão constante, 2) modo de reatância constante, e 3) modo de potência constante. Enquanto os dois primeiros modos acima citados são usados para realizar apenas compensação série de potência reativa, o modo de potência constante também permite que o fluxo de potência na rede de transmissão seja controlado. Uma vez que esses modos podem causar diferentes impactos na estabilidade angular do sistema, o objetivo desse trabalho é investigar a influência do modo de operação do SSSC na estabilidade de ângulo a pequenas perturbações e na estabilidade transitória de sistemas de potência. O estudo é baseado na técnica de análise modal bem como em simulações no domínio do tempo, as quais são realizadas usando o PSAT (Power System Analysis Toolbox). Os resultados obtidos mostram que, quando realizando apenas compensação série de potência reativa, o uso do SSSC no modo de reatância constante é a melhor estratégia para melhorar a estabilidade de ângulo a pequenas perturbações bem como a estabilidade transitória.

PALAVRAS-CHAVE: estabilidade de ângulo a pequenas perturbações, estabilidade transitória, FACTS, PSAT, SSSC. 


\section{INTRODUÇÃO}

Devido às políticas de desregulamentação, empresas do setor elétrico por todo mundo estão operando em mercados cada vez mais competitivos. Ao mesmo tempo, pressões econômicas e ambientais impõem severas restrições à construção de novas linhas de transmissão, de forma que o aumento da capacidade de transferência de potência dos sistemas é, usualmente, conseguido pelo reforço das linhas já existentes. O desenvolvimento de novas tecnologias, tal como os FACTS (Flexible AC Transmission System), tem ajudado as empresas do setor elétrico a lidarem com os problemas acima mencionados. Além de estenderem os limites de transmissão da rede, controladores FACTS podem exercer controle contínuo sobre diversas grandezas elétricas, tais como impedâncias e tensões, e, assim, também contribuem de forma expressiva para a melhoria da estabilidade dos sistemas (Song e Johns, 1999), (Hingorani e Gyugyi, 2000).

Dentre os controladores FACTS empregados para compensação série de potência reativa, os mais abordados na literatura são o TCSC (Thyristor Controlled Series Capacitor) e o SSSC (Static Synchronous Series Compensator). Para fornecer ou absorver potência reativa, um TCSC utiliza bancos de capacitores e reatores. Estudos relatando aplicações do TCSC para melhorar o desempenho de sistemas são apresentados em (Martins et al., 1999), (Del Rosso et al., 2003). Atualmente existem diversos controladores TCSC em operação. No Brasil, dois controladores TCSC foram instalados na linha de interligação Norte-Sul com o principal objetivo de amortecer as oscilação eletromecânicas do modo interárea do sistema interligado brasileiro (Gama, 1999).

Em vez de banco de capacitores e reatores, um SSSC utiliza conversores fonte de tensão ou corrente, baseados em chaves auto-comutadas, para produzir tensão trifásica em quadratura com a corrente da linha de transmissão (Song e Johns, 1999). Neste trabalho, optou-se por representar conversores fonte de tensão, visto que por razões econômicas e de desempenho, estes são mais indicados para aplicações com FACTS (Hingorani e Gyugyi, 2000). Ainda não existem controladores SSSC operando isoladamente, mas sim como a parte série do controlador série-paralelo UPFC (Unified Power Flow Controller) (Mehraban et al., 1998). Para atingir específicos objetivos de compensação, a tensão série do SSSC pode ser controlada de diversas formas. Os modos de controle do SSSC mais discutidos na literatura são (Hingorani e Gyugyi, 2000),(Zhang, 2003): 1) modo de tensão constante, no qual a tensão do SSSC é mantida constante independentemente da corrente na linha, 2) modo de reatância constante, no qual a tensão do SSSC é proporcional à corrente na linha, e 3) modo de potência constante, no qual a tensão do SSSC é usada para regular o fluxo de potência na rede de transmissão. Em qualquer um desses modos, contro-

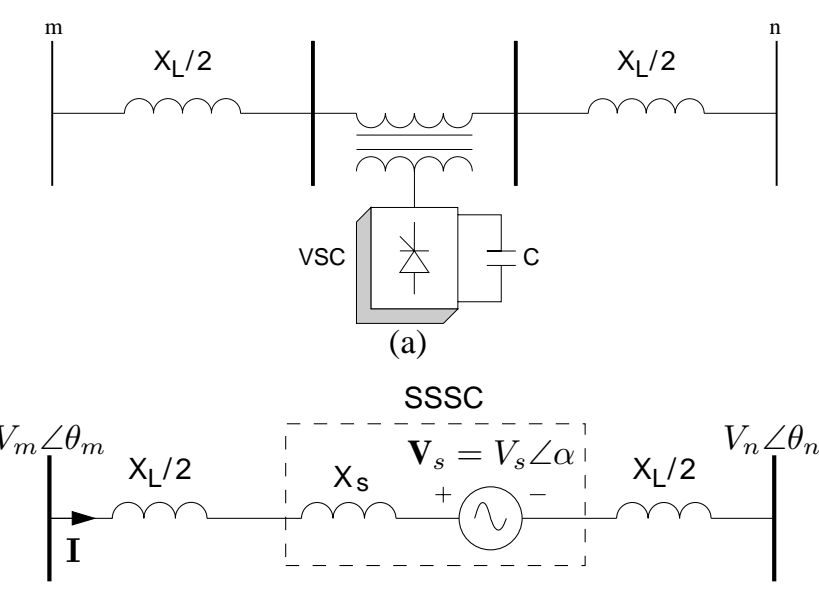

(b)

Figura 1: SSSC conectado entre as barras $m$ e $n$; (a) diagrama unifilar, (b) circuito equivalente.

ladores suplementares, tal como para amortecimento de oscilações, podem ser usados para melhorar o comportamento dinâmico do sistema (Hingorani e Gyugyi, 2000).

O objetivo desse trabalho é investigar a influência do modo de operação do SSSC na estabilidade de ângulo a pequenas perturbações e na estabilidade transitória (grandes perturbações) de sistemas elétricos de potência. Os estudos são baseados na técnica de análise modal bem como em simulação digital no domínio do tempo, as quais foram realizadas usando o PSAT (Power System Analysis Toolbox) (Milano, 2005). O PSAT é um toolbox do MATLAB ${ }^{\circledR}$ para análise estática, dinâmica, bem como para estudos que abordem controle de sistemas elétricos de potência. Ele possui um ampla biblioteca e contempla fluxo de carga, fluxo de carga da continuação, fluxo de carga ótimo, análise de estabilidade a pequenas perturbações e simulação dinâmica no domínio do tempo. Uma descrição mais detalhada do PSAT pode ser encontrada em (Milano, 2005).

\section{MODELAGEM E CONTROLE DO SSSC}

Em função dos modelos de controladores FACTS disponibilizados na biblioteca das primeiras versões do PSAT apresentarem problemas de convergência, fez-se necessária a inclusão de um modelo apropriado do SSSC para os estudos desenvolvidos neste trabalho. Tal modelagem é detalhadamente descrita nesta seção e foi submetida e incorporada à biblioteca oficial do PSAT por seu mantenedor (Milano, 2006).

Na Figura 1(a) é mostrado um SSSC conectado ao sistema entre as barras $m$ e $n$. O circuito equivalente é mostrado na Figura 1(b), no qual o SSSC é representado por uma fonte de tensão $\mathbf{V}_{s}$ acompanhada pela reatância de dispersão $X_{s}$ do 


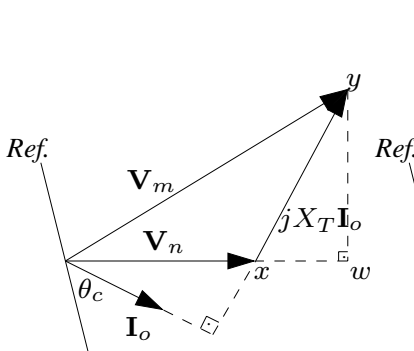

(a)

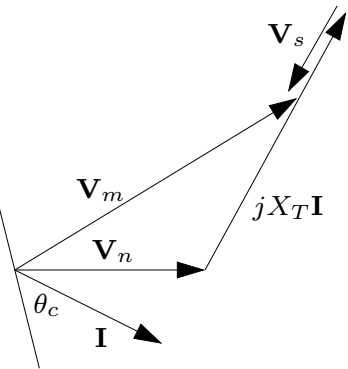

(b)

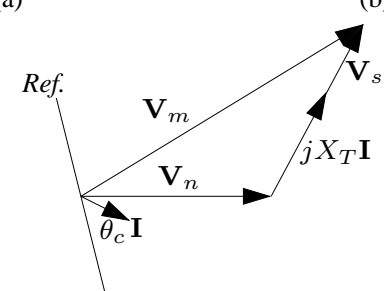

(c)

Figura 2: Diagrama fasorial; (a) $\mathbf{V}_{\mathbf{s}}=0$, (b) $\mathbf{V}_{\mathbf{s}}$ atrasada $90^{\circ}$ de $\mathbf{I}$, (c) $\mathbf{V}_{\mathbf{s}}$ adiantada $90^{\circ}$ de $\mathbf{I}$.

transformador de acoplamento. O parâmetro controlável do SSSC é $V_{s}$, que representa a magnitude da tensão $\mathbf{V}_{s}$. A Figura 2 mostra o diagrama fasorial do sistema, considerando várias condições de operação do SSSC. Observe que a tensão $\mathbf{V}_{s}$ altera somente a magnitude da corrente na linha $I$, mas não seu ângulo $\theta_{c}$ (Kumkratug e Haque, 2003). Quando $\mathbf{V}_{s}=0$ (Figura 2(a)), a corrente na linha é dada por:

$$
\mathbf{I}_{o}=\frac{\mathbf{V}_{m}-\mathbf{V}_{n}}{j X_{T}}
$$

sendo $X_{T}=X_{L}+X_{s}$. O ângulo da corrente pode ser escrito como:

$$
\theta_{c}=\tan ^{-1}\left[\frac{V_{n} \cos \theta_{n}-V_{m} \cos \theta_{m}}{V_{m} \sin \theta_{m}-V_{n} \sin \theta_{n}}\right]
$$

Da Figura 2(b), a equação (1) pode ser generalizada como:

$$
\begin{gathered}
\mathbf{I}=\frac{\mathbf{V}_{m}-\mathbf{V}_{s}-\mathbf{V}_{n}}{j X_{T}}= \\
{\left[\frac{\mathbf{V}_{m}-\mathbf{V}_{n}}{j X_{T}}\right]+\left[-\frac{\mathbf{V}_{s}}{j X_{T}}\right]=\mathbf{I}_{o}+\Delta \mathbf{I}}
\end{gathered}
$$

sendo $\Delta \mathbf{I}$ o termo adicional de corrente devido à tensão $\mathbf{V}_{s}$. O fluxo de potência da barra $m$ para a barra $n$ pode ser escrito como:

$$
\mathbf{S}_{m n}=\mathbf{V}_{m} \mathbf{I}^{*}=\mathbf{S}_{m n_{o}}+\Delta \mathbf{S}_{m n}
$$

na qual $S_{m n_{o}}$ representa o fluxo de potência para $\mathbf{V}_{s}=0$. As partes real e imaginária do termo adicional de potência
$\Delta \mathbf{S}_{m n}$ devido à tensão do SSSC são dadas, respectivamente, por:

$$
\Delta P_{m n}=\Re_{e}\left\{\mathbf{V}_{m} \Delta \mathbf{I}^{*}\right\}=\frac{V_{m} V_{s}}{X_{T}} \sin \left(\theta_{m}-\alpha\right)
$$

$\mathrm{e}$

$$
\Delta Q_{m n}=\Im_{m}\left\{\mathbf{V}_{m} \Delta \mathbf{I}^{*}\right\}=-\frac{V_{m} V_{s}}{X_{T}} \cos \left(\theta_{m}-\alpha\right)
$$

Para a tensão $\mathbf{V}_{s}$ atrasada da corrente por $90^{\circ}\left(\alpha=\theta_{c}-90^{\circ}\right)$, (5) e (6) podem ser reescritas como segue:

$$
\Delta P_{m n}=\frac{V_{m} V_{s}}{X_{T}} \cos \left(\theta_{m}-\theta_{c}\right)
$$

$$
\Delta Q_{m n}=\frac{V_{m} V_{s}}{X_{T}} \sin \left(\theta_{m}-\theta_{c}\right)
$$

Da equação (2), o termo $\cos \left(\theta_{m}-\theta_{c}\right)$ na equação (7) é dado por:

$$
\cos \left(\theta_{m}-\theta_{c}\right)=\frac{V_{n}}{V_{m}} \cos \left(\theta_{n}-\theta_{c}\right)
$$

e da Figura 2(a) tem-se que:

$$
\cos \left(\theta_{n}-\theta_{c}\right)=\frac{y w}{x y}
$$

sendo

$$
y w=V_{m} \sin \left(\theta_{m n}\right)
$$

$\mathrm{e}$

$$
x y=\sqrt{V_{m}^{2}+V_{n}^{2}-2 V_{m} V_{n} \cos \theta_{m n}}
$$

nas quais $\theta_{m n}=\theta_{m}-\theta_{n}$.

Usando as equações (7), (8), e (10) combinadas com (11) e (12), os termos $\Delta P_{m n}$ e $\Delta Q_{m n}$ podem ser expressos por:

$$
\begin{aligned}
& \Delta P_{m n}= \frac{V_{m} V_{n}}{X_{T}} \sin \theta_{m n} \times \\
& \frac{V_{s}}{\sqrt{V_{m}^{2}+V_{n}^{2}-2 V_{m} V_{n} \cos \theta_{m n}}} \\
& \Delta Q_{m n}=-\frac{V_{m}\left(V_{n} \cos \theta_{m n}-V_{m}\right)}{X_{T}} \times \\
& \frac{V_{s}}{\sqrt{V_{m}^{2}+V_{n}^{2}-2 V_{m} V_{n} \cos \theta_{m n}}}
\end{aligned}
$$


e, finalmente, os fluxos de potência ativa e reativa entre as barras $m$ e $n$, nos dois sentidos, são dados por:

$$
\begin{aligned}
P_{m n}= & -P_{n m}=\frac{V_{m} V_{n}}{X_{T}} \sin \theta_{m n} \times \\
& \left(1+\frac{V_{s}}{\sqrt{V_{m}^{2}+V_{n}^{2}-2 V_{m} V_{n} \cos \theta_{m n}}}\right) \\
Q_{m n}= & \frac{V_{m}}{X_{T}}\left(V_{m}-V_{n} \cos \theta_{m n}\right) \times \\
& \left(1+\frac{V_{s}}{\sqrt{V_{m}^{2}+V_{n}^{2}-2 V_{m} V_{n} \cos \theta_{m n}}}\right) \\
Q_{n m}= & \frac{V_{n}}{X_{T}}\left(V_{n}-V_{m} \cos \theta_{m n}\right) \times \\
& \left(1+\frac{V_{s}}{\sqrt{V_{m}^{2}+V_{n}^{2}-2 V_{m} V_{n} \cos \theta_{m n}}}\right)
\end{aligned}
$$

Observe que, se $V_{s}>0$, o SSSC opera realizando compensação capacitiva. De modo contrário, se $V_{s}<0$, compensação indutiva é realizada.

\subsection{Sistema de Controle}

O sistema de controle de um SSSC é composto por um controle interno e por um controle externo. O controle interno fornece sinais de disparo e corte apropriados para os tiristores GTO (Gate Turn-Off) de forma que o conversor estático produza a tensão de compensação desejada. O controle externo define a referência para o controle interno de modo que o objetivo de compensação especificado seja realizado. Além disso, uma malha de controle suplementar pode ser incluída no controle externo. O diagrama do sistema de controle de um SSSC é mostrado na Figura 3. Nesta figura, $V_{c}$ representa o valor da tensão de compensação, o qual é determinado pela malha de controle de compensação, e $V_{e}$ representa o valor de saída da malha de controle de estabilidade. O valor final da magnitude da tensão ordenado pelo controle externo é $V_{s}^{\prime}$, dado em qualquer instante pela soma de $V_{c}$ e $V_{e}$. Em condições de regime permanente, o valor de $V_{s}^{\prime}$ é determinado somente pela malha de controle de compensação, uma vez que $V_{e}$ é nula. O controle interno e o atraso de resposta do conversor são representados por um bloco de primeira ordem, cuja saída é a magnitude da tensão série inserida pelo SSSC, a qual é sempre mantida entre seus limites máximo e mínimo. Embora o modelo apresentado não inclua representação detalhada dos dispositivos eletrônicos, o mesmo é adequado para estudos nos quais o foco não está na dinâmica do conversor (muito mais rápida do que a dinâmica dos geradores), mas sim nos transitórios eletromecânicos dos geradores, como neste trabalho.

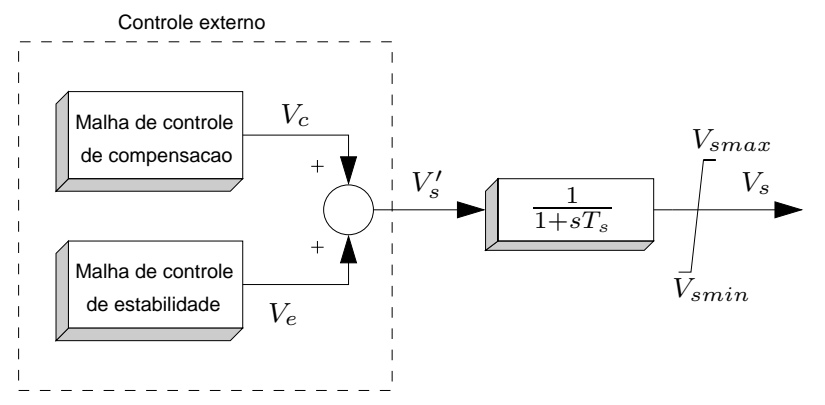

Figura 3: Diagrama do sistema de controle externo do SSSC.

\subsection{Malha de Controle de Compensação}

O modo de operação do SSSC é determinado pela malha de controle de compensação. Os modos de controle estudados nesse trabalho são: 1) modo de tensão constante; 2) modo de impedância constante; e 3) modo de potência constante. No modo de tensão constante, o controle interno do SSSC atua para manter a tensão $V_{s}$ constante em um valor especificado $\left(V_{c}=\right.$ cte). Observe que, neste modo, mesmo em situações nas quais a magnitude da corrente na linha é pequena, uma considerável quantidade de potência reativa pode ser injetada/absorvida em série com a linha de transmissão, dependendo do magnitude e defasagem de $\mathbf{V}_{\mathbf{s}}$. No modo de reatância constante, a tensão do SSSC varia proporcionalmente à magnitude da corrente na linha de forma que a reatância efetiva da linha de transmissão seja mantida constante. Neste modo, o valor de $V_{c}$ é dado por:

$$
V_{c}=k X_{T} I
$$

sendo $k$ o nível de compensação série especificado. Assim, $k X_{T}=X_{S S S C}$ representa a reatância série que produziria a mesma queda de tensão que o SSSC. Após algumas manipulações algébricas, (18) pode ser escrita como:

$$
V_{c}=\frac{k}{1-k} \sqrt{V_{m}^{2}+V_{n}^{2}-2 V_{m} V_{n} \cos \theta_{m n}}
$$

com $0 \leq k<1$. Diferentemente dos dois primeiros modos de operação já descritos, nos quais o objetivo é realizar somente compensação série de potência reativa, no modo de potência constante o SSSC, por meio da injeção/obsorção de potência reativa, é usado para manter o fluxo de potência ativa na rede em rotas de transmissão especificadas. Isto pode ser conseguido manualmente por meio da intervenção do operador, ou automaticamente por meio de um controlador proporcional-integrador (PI) cuja entrada deve ser a diferença entre o valor de referência e o valor medido, conforme mostrado na Figura 4. Assim, um SSSC pode ser usado para manter constante o fluxo de potência ativa na linha compensada, ou para redirecionar as transferências de potência entre sistemas interligados. 


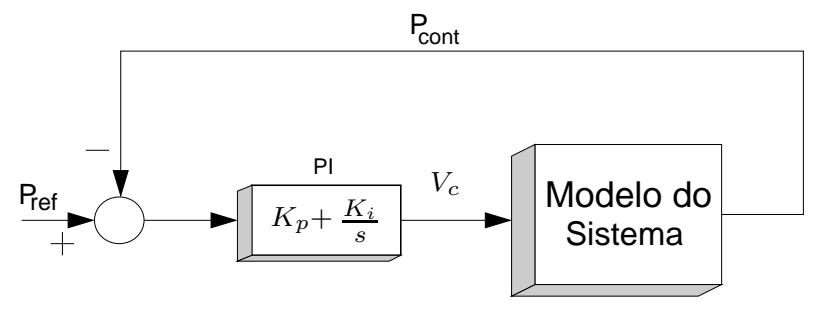

Figura 4: Diagrama de controle do SSSC no modo de potência constante.

\subsection{Malha de Controle de Estabilidade}

Além de executar sua função de compensação, ditada pela malha de controle de compensação, um SSSC também pode estender os limites de estabilidade do sistema por meio de sua malha de controle de estabilidade (ver Figura 3). Em (Duangkamol et al., 2000) é apresentada uma estratégia para limitar a corrente de falta e melhorar a estabilidade de regime permanente do sistema. Aqui, um controlador é usado para modular a tensão do SSSC durante transitórios com o objetivo de amortecer oscilações eletromecânicas. Tal controlador, denominado de controlador POD (Power Oscillation Damping), é formado por três blocos; um filtro passa-alta, um circuito compensador de fase e um ganho, conforme mostrado na Figura 5. O filtro passa-alta é usado para evitar que o estabilizador atue em regime permanente. O compensador de fase fornece a característica de fase apropriada ao sinal de entrada, e o ganho determina a quantidade de amortecimento introduzida pelo estabilizador.

A escolha de um sinal de entrada adequado é de fundamental importância para o projeto de um controlador efetivo e robusto. A seguir são listadas as principais características de um sinal de entrada apropriado (Watanabe et al., 1998),(Del Rosso et al., 2003):

- O sinal de entrada deve estar disponível localmente ou deve poder ser sintetizado a partir de medidas locais. A utilização de um sinal local elimina a necessidade do uso de canais de comunicação, reduzindo atrasos de resposta, aumentando a confiabilidade e diminuindo os custos de implementação do controlador.

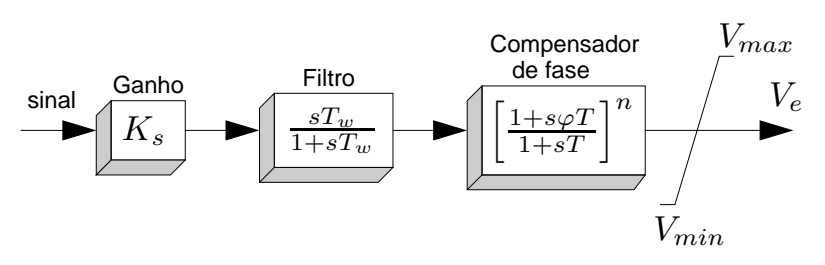

Figura 5: Controlador POD.
- Objetivando fornecer amortecimento adequado, o sinal de entrada deve observar o modo a ser amortecido;

- O sinal de entrada deve fornecer ações de controle corretas em resposta a ocorrência de uma perturbação severa.

Muitas técnicas podem ser usadas para o ajuste dos parâmetros do controlador para amortecimento de oscilações de equipamentos FACTS. Neste trabalho o ajuste da malha de controle de estabilidade do SSSC é realizado por meio do método da resposta em frequência baseado nos conceitos de margem de fase e margem de ganho. Dois possíveis candidatos, fluxo de potência ativa e magnitude de corrente, são considerados como sinais de entrada do controlador POD e seus desempenhos são investigados.

\section{SISTEMA TESTE}

Para investigar a influência do modo de operação do SSSC na estabilidade angular, o sistema mostrado na Figura 6 foi considerado nos estudos. Esse sistema compreende uma planta de geração termoelétrica formada por quatro geradores de 555 MVA, $24 \mathrm{kV}, 60 \mathrm{~Hz}$, conectada à um barramento infinito por meio de um transformador elevador seguido de duas linhas de transmissão (Kundur, 1994). Um gerador com modelo de sexta ordem é usado para representar a planta. Tal gerador é equipado com um regulador automático de tensão representado pelo AVR tipo III da biblioteca do PSAT. Um SSSC está conectado entre as barras 2 e 3 compensando a linha de transmissão mais fraca do sistema (linha 2-3-4). Os dados do sistema são fornecidos no Apêndice.

Com o intuito de propiciar uma melhor compreensão das diferenças entre os modos de operação do SSSC, nesta seção são apresentadas respostas do sistema à aplicação de um degrau de $10 \%$ na potência mecânica do gerador considerando o SSSC operando de diferentes formas. Embora respostas ao degrau são usualmente obtidas por meio do modelo linearizado do sistema, os resultados apresentados foram obtidos considerando-se o modelo não linearizado do sistema. O ponto de operação considerado corresponde a uma situação de carregamento elevado na qual a planta está entregando 1.998 MW (ou 0,9 p.u.) ao barramento infinito. Para esta condição, a tensão do SSSC faz com que o valor da impedância efetiva da linha 2-3-4 seja o mesmo da linha 2-4 de modo que o fluxo de potência seja igualmente distribuído entre as duas linhas de transmissão.

Caso (A): o SSSC opera no modo de tensão constante. A Figura 7 mostra os desvios de fluxo de potência no transformador e nas linhas 2-4 e 2-3-4. Observa-se que o valor final do fluxo de potência na linha 2-4 é maior do que na linha 2-34. Isto ocorre porque após a perturbação a impedância efetiva 


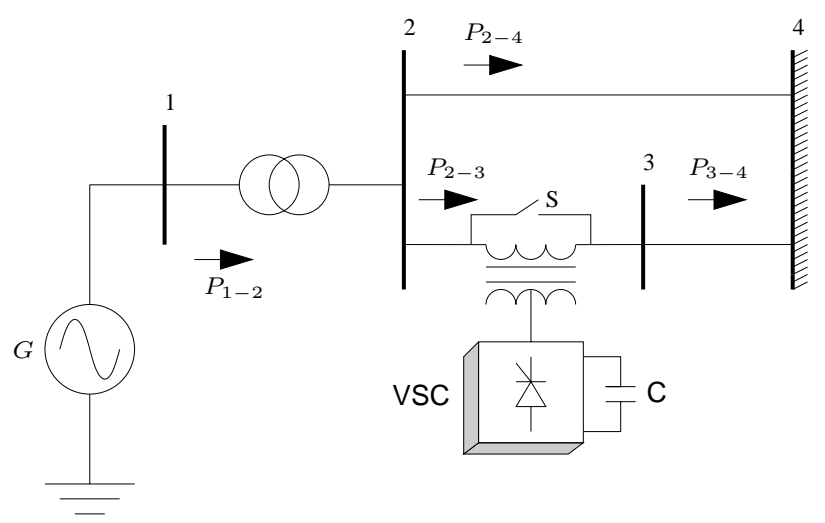

Figura 6: Sistema teste com SSSC.

da linha compensada é maior do que a impedância da linha não compensada, uma vez que a tensão do SSSC é mantida constante não compensando o acréscimo da queda de tensão na linha causado pelo acréscimo na geração. Verifica-se também que as oscilações de fluxo de potência na rede de transmissão são pobremente amortecidas.

Caso (B): o SSSC opera no modo de reatância constante. Na Figura 8 são mostrados os desvios de fluxos de potência na rede. Nota-se que, neste caso, o acréscimo na geração é igualmente dividido entre as duas linhas do corredor de transmissão, uma vez que o valor da impedância das duas linhas (2-3-4 e 2-4) é o mesmo em qualquer instante devido a ação da malha de controle de compensação do SSSC.

Caso (C): o SSSC opera no modo de potência constante. A estratégia de controle escolhida atua para manter constante o fluxo de potência na linha compensada $\left(\mathrm{P}_{\text {cont }}=P_{2-3}\right)$. O projeto do controlador PI foi baseado na técnica de alocação

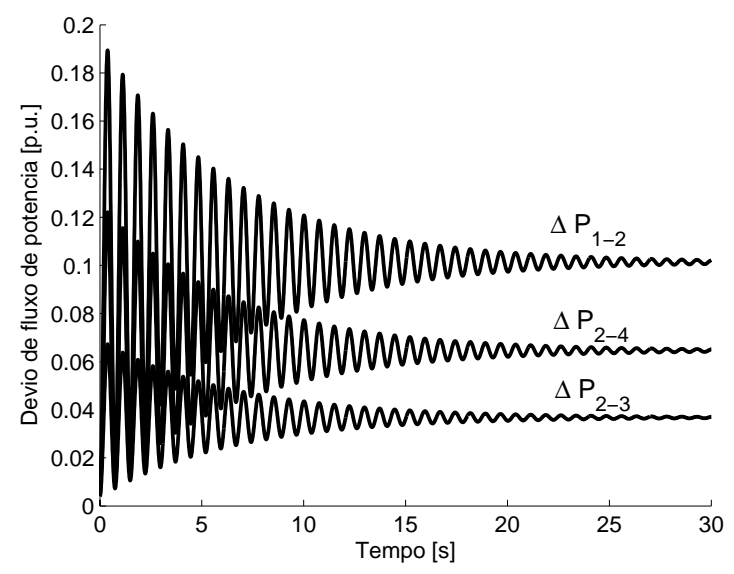

Figura 7: Desvio de fluxo de potência - Caso (A)

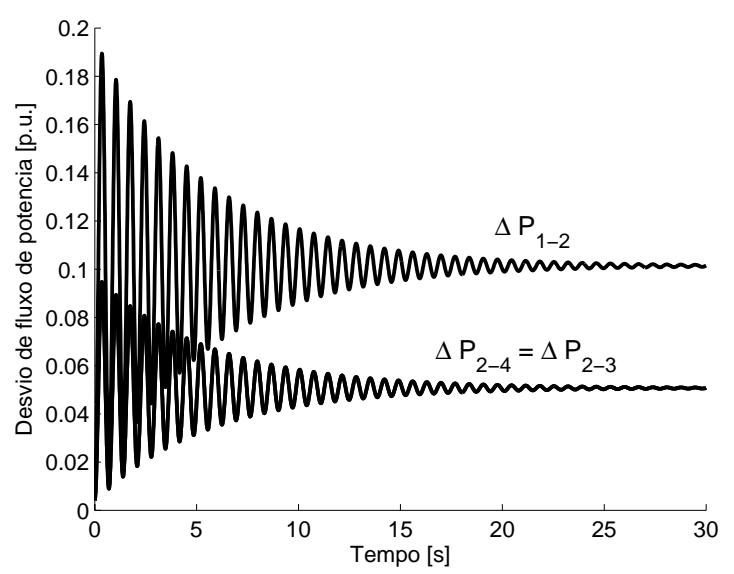

Figura 8: Desvio de fluxo de potência - Caso (B)

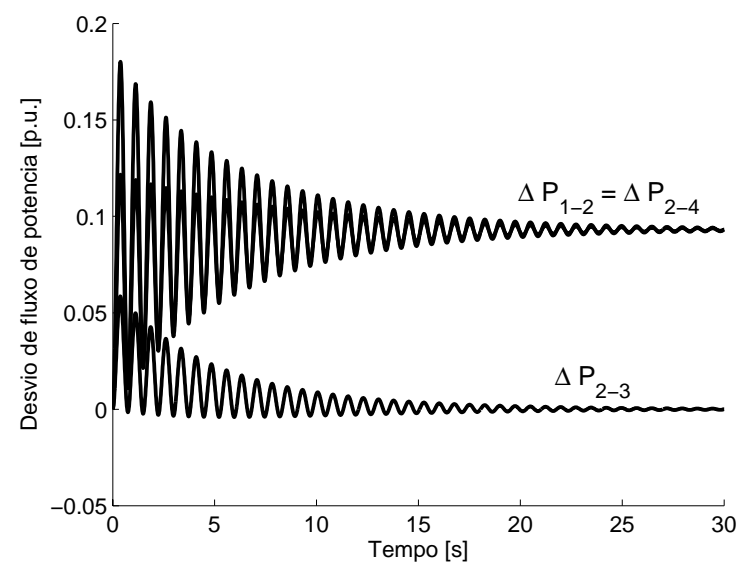

Figura 9: Desvio de fluxo de potência - Caso (C)

de pólos e os ganhos $K_{i}$ e $K_{p}$ foram selecionados de forma a causar um mínimo impacto no modo eletromecânico. A Figura 9 mostra a linha 2-4 absorvendo todo acréscimo de geração (10\%). Nota-se que o fluxo de potência na linha 23 retorna ao seu valor preestabelecido por meio da ação do controlador PI. Observa-se também que o PI é de ação lenta, a qual é finalizada somente após $25 \mathrm{~s}$.

Caso (D): o SSSC opera no modo de potência constante atuando para manter constante o fluxo de potência na linha 2-4 $\left(\mathrm{P}_{\text {cont }}=P_{2-3}-P_{1-2}\right)$, de forma que a linha compensada absorva todo o acréscimo de geração, conforme apresentado na Figura 10. Neste caso é mostrado que o SSSC pode controlar o fluxo de potência em linhas nas quais o mesmo não se encontra instalado. Nota-se que, para realização dessa função, foi necessário utilizar o sinal do fluxo de potência no transformador $\left(P_{1-2}\right)$. Uma vez que o SSSC está instalado próximo da barra 2 , esse sinal pode ser considerado um sinal 


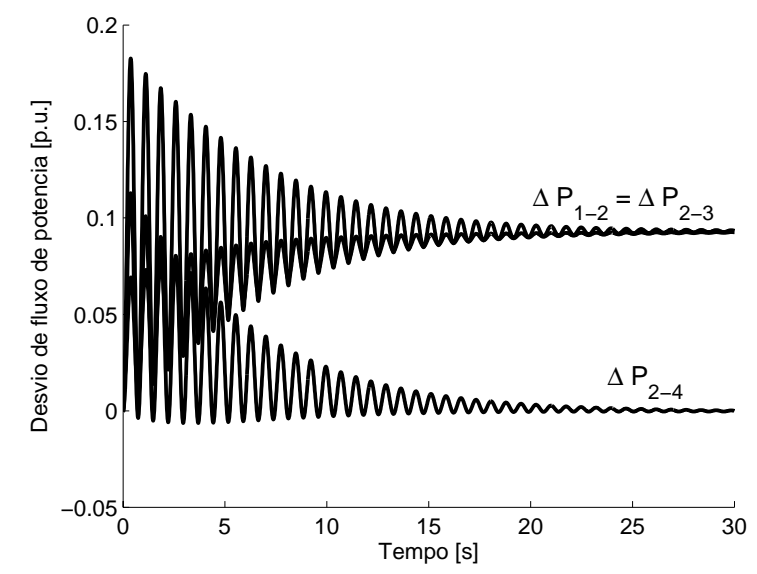

Figura 10: Desvio de fluxo de potência - Caso (D)

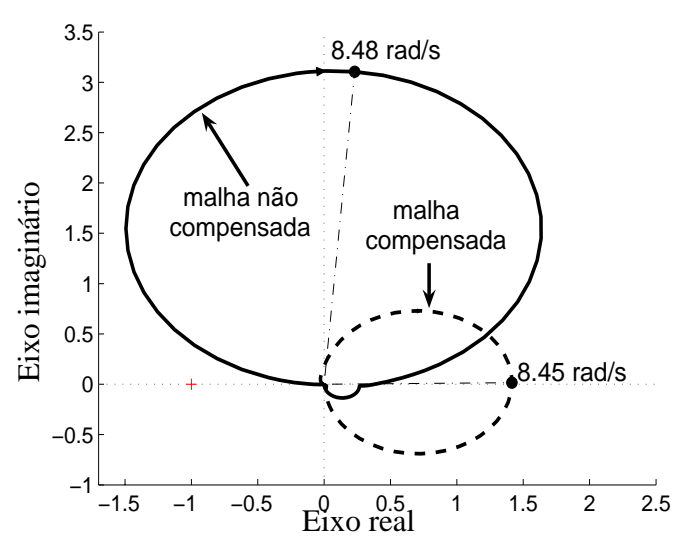

Figura 11: Gráficos de Nyquist.

local.

Em todos os casos apresentados até agora, o modo eletromecânico é pobremente amortecido $(\zeta<5 \%)$ e, por isso, as oscilações de fluxo de potência na rede de transmissão desaparecem somente após um longo tempo (maior que $20 \mathrm{~s}$ ). Nos Casos (E) e (F) é investigada a eficiência do SSSC para realizar de forma conjunta o controle de fluxo de potência e o amortecimento de oscilações. Então, além do controlador PI na malha de controle de compensação, o SSSC deve ser equipado com o controlador POD em sua malha de controle de estabilidade (ver Figura 5). O PI é usado para manter constante o fluxo de potência na linha compensada, como no Caso (C). Conforme anteriormente mencionado, dois candidatos para sinal de entrada da malha de controle de estabilidade são considerados: o fluxo de potência ativa e a magnitude da corrente no transformador $\left(P_{2-1}\right.$ e $\left.I_{2-1}\right)$.
Tabela 1: Parâmetros dos controladores POD

\begin{tabular}{cccccc}
\hline $\begin{array}{c}\text { Sinal } \\
\text { de entrada }\end{array}$ & $\begin{array}{c}K_{s} \\
\text { (p.u./p.u.) }\end{array}$ & $\begin{array}{c}T_{w} \\
(\mathrm{~s})\end{array}$ & $\begin{array}{c}T \\
(\mathrm{~s})\end{array}$ & & $\varphi$ \\
\hline$P_{2-1}$ & 1.280 & 3 & 0.280 & 0.175 & 2 \\
$I_{2-1}$ & 0.038 & 3 & 0.049 & 5.918 & 2 \\
\hline
\end{tabular}

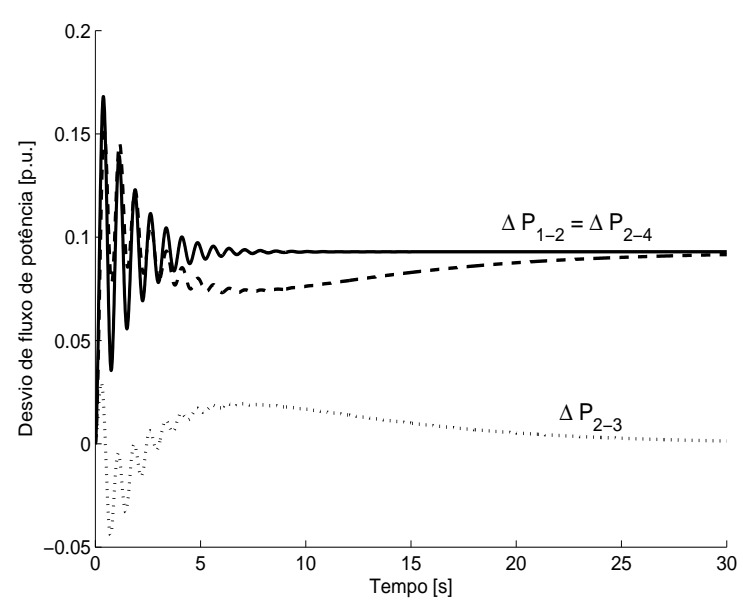

Figura 12: Desvio de fluxo de potência - Caso (E)

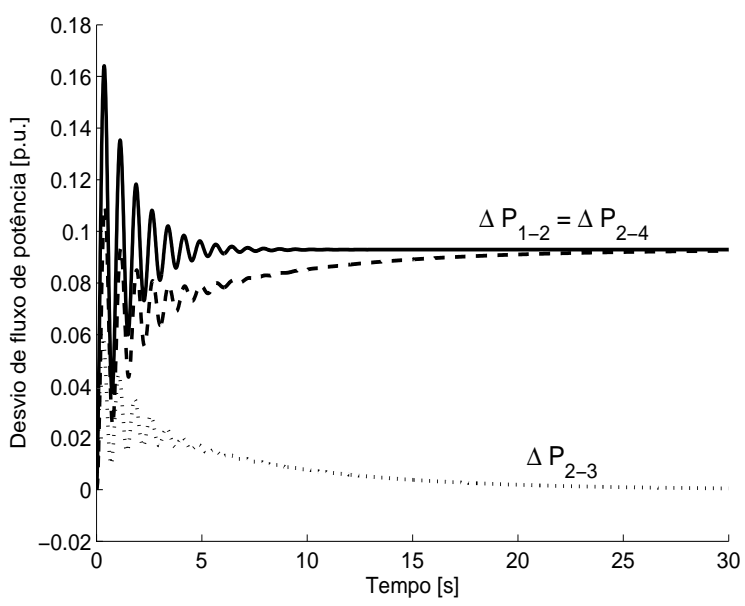

Figura 13: Desvio de fluxo de potência - Caso (F)

A técnica usada neste trabalho para o projeto do controlador POD é baseada no gráfico de Nyquist de uma função de transferência do sistema em malha aberta. Quando a potência ativa no transformador é considerada como sinal de entrada, a função de transferência a ser analisada é $\Delta P_{2-1}(s) / \Delta V_{e}(s)$. A Figura 11 mostra o gráfico de Nyquist da função de transferência não compensada bem como da função de transferência compensada pelo estabilizador derivado do sinal da potência (somente frequências positivas são mostradas). Nesse caso (sistema estável em malha aberta), a fase a ser compensada é calculada de forma que o diagrama 
de Nyquist da função de transferência compensada fique o mais distante possível do ponto de instabilidade $(-1,0)$, garantindo boa margem de fase para o sistema em malha fechada. Assim, o centro da frequência de 8,48 rad/s é realocado para o eixo real por meio de um atraso de aproximadamente $90^{\circ}$. Em seguida, o ganho do estabilizador é ajustado para se obter a taxa de amortecimento desejada. Este mesmo procedimento também foi utilizado para projetar o estabilizador baseado no sinal $I_{2-1}$ e os parâmetros dos dois controladores são fornecidos na Tabela 1. Maiores informações sobre a técnica de projeto usada neste trabalho podem ser obtidas em (Martins e Lima, 1990) e (Elices et al., 2004).

Caso (E): o SSSC está equipado com o estabilizador derivado do sinal $P_{2-1}$. Observa-se na Figura 12 que, apesar do amortecimento adequado, existem picos elevados nas respostas transitórias de $P_{2-4}$ e $P_{2-3}$. Esses picos são causados pela presença de zeros próximos à origem para as funções de transferência $P_{2-4} / P_{m e c}(\mathrm{z}=-0.001)$ e $P_{2-3} / P_{m e c}(\mathrm{z}=$ $0.003)$. Além de causar picos excessivos na resposta transitória do sistema, zeros próximos à origem podem prejudicar a estabilidade do sistema, conforme discutido em (Martins e Lima, 1990) e (Martins et al., 1999).

Caso (F): o SSSC está equipado com o estabilizador derivado do sinal $I_{2-1}$. A eficiência de tal controlador pode ser avaliada por meio da resposta ao degrau mostrada na Figura 13. Neste caso, a funções de transferência $P_{2-4} / P_{m e c}$ e $P_{2-3} / P_{m e c}$ não possuem zeros próximos à origem e, assim, $P_{2-4}$ e $P_{2-3}$ apresentam respostas transitórias adequadas, além de serem devidamente amortecidas. Por isso, o sinal da magnitude da corrente é um melhor candidato do que o sinal da potência para ser usado como sinal de entrada da malha de controle de estabilidade do SSSC.

\section{ANÁLISE DA ESTABILIDADE A PEQUE- NAS PERTURBAÇÕES}

A investigação da influência do modo de operação do SSSC na estabilidade de ângulo a pequenas perturbações é realizada considerando-se o modelo linearizado do sistema ilustrado na Figura 6. Por meio dessa linearização, a técnica de análise modal é aplicada e informações sobre os torques sincronizante e de amortecimento podem ser obtidas. Tais torques são associados, respectivamente, à parte imaginária e à parte real dos autovalores relacionados ao desvio de velocidade e do ângulo do rotor $(\Delta \omega$ e $\Delta \delta$ ), ou seja, ao modo eletromecânico (Kundur, 1994).

Nesta e na próxima seção, o valor do nível de compensação série realizado pelo SSSC (valor de $k$ ) é sempre determinado no carregamento de 0,5 p.u. (ou 1.110 MW). Em todos cenários estudados o SSSC está equipado com o POD usado no Caso (F) da seção anterior. Primeiramente, é feita uma com-

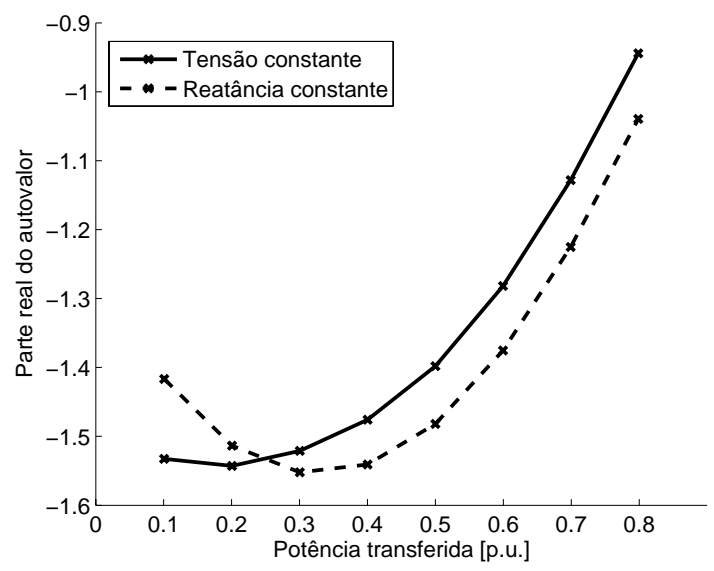

Figura 14: Trajetória da parte real do modo eletromecânico.

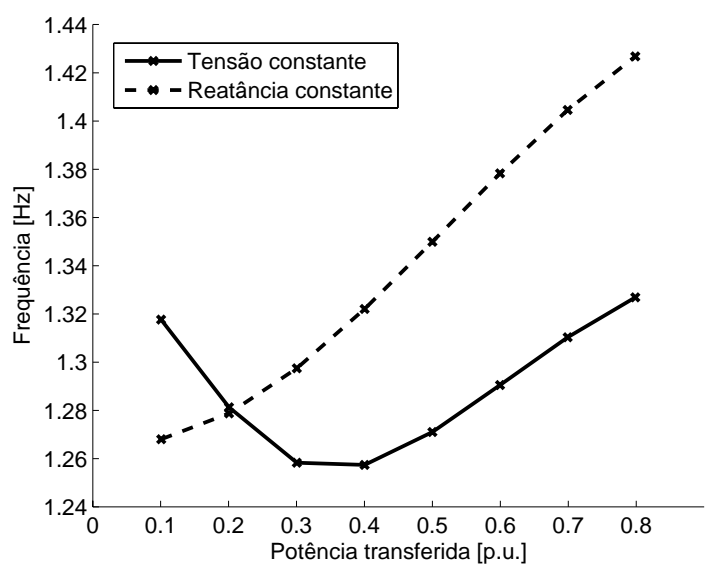

Figura 15: Frequência do modo eletromecânico.

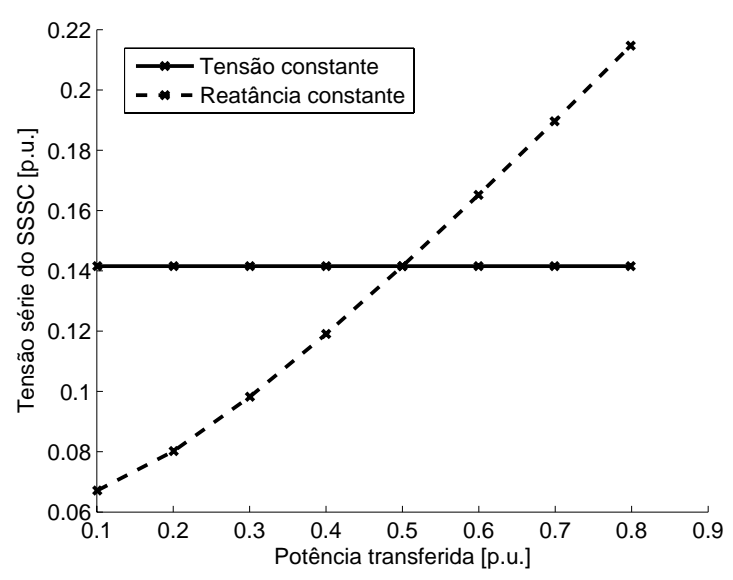

Figura 16: Tensão série do SSSC. 


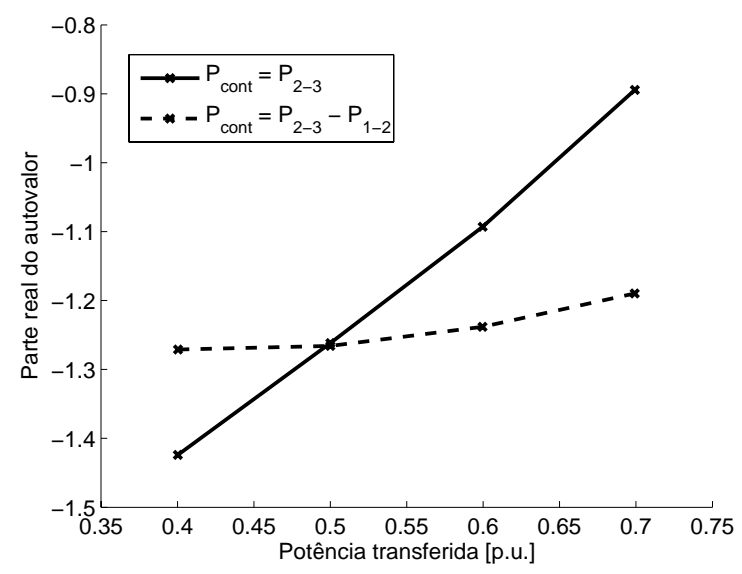

Figura 17: Trajetória da parte real do modo eletromecânico.

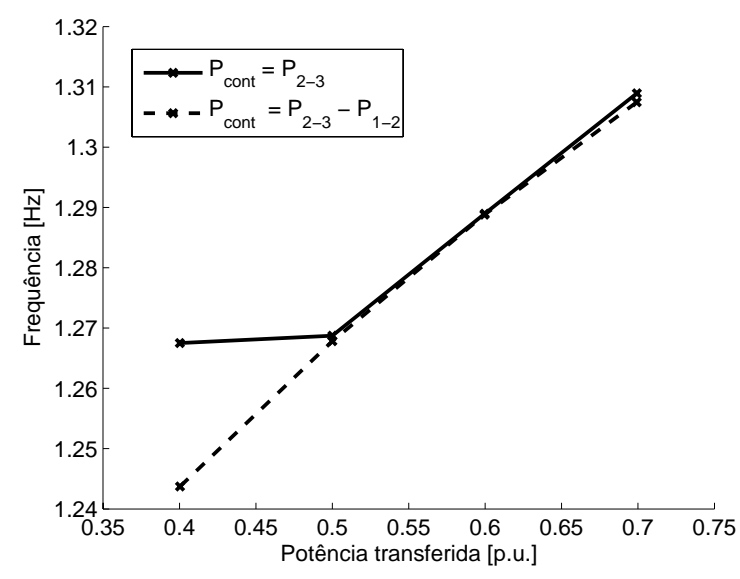

Figura 18: Frequência do modo eletromecânico.

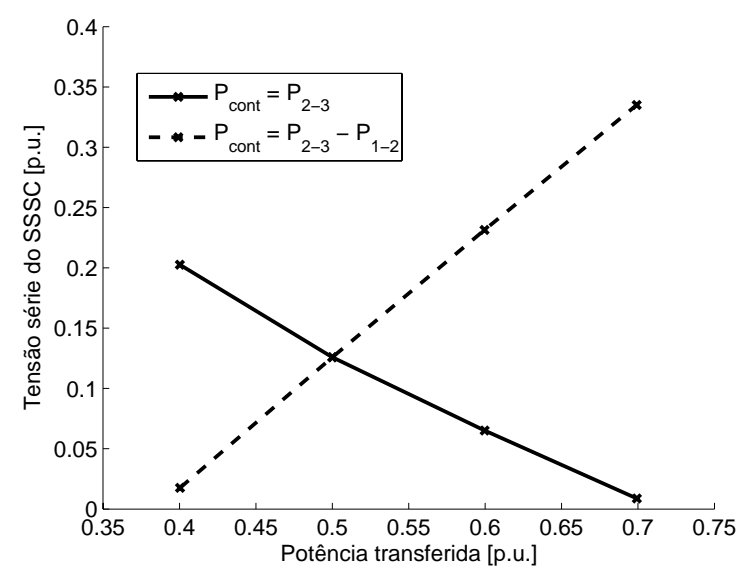

Figura 19: Tensão série do SSSC. paração entre os modos de operação usados para compensação série de potência reativa. Em seguida, as duas estratégias para controle de fluxo de potência descritas neste trabalho também são analisadas.

As Figuras 14, 15, e 16 mostram, respectivamente, as trajetórias da parte real, da parte imaginária (frequência) do modo eletromecânico e o comportamento da tensão série do SSSC em função do carregamento do sistema, considerando-se os modos de tensão e reatância constante. Para obtenção destas curvas foi usado um nível de compensação série de 50\%, ou seja, $k=0,5$. Isto implica que, no carregamento de 0,5 p.u., o valor da tensão do SSSC é o mesmo nos dois modos de operação (ver Figura 16). É observado que, mesmo com uma menor tensão, o SSSC operando no modo de reatância constante fornece maiores torques de amortecimento e sincronizante. Exceção é feita para condições de carregamento leve (menor que 0,25 p.u.) em que a tensão aplicada pelo modo de tensão constante é bem maior que a tensão do modo de reatância constante. Estes resultados permitem concluir que um SSSC de menor capacidade, operando no modo de reatância constante, pode fornecer maior quantidade de torques sincronizante e de amortecimento do que um SSSC de maior capacidade operando no modo de tensão constante.

O comportamento do modo eletromecânico e da tensão série do SSSC em função do carregamento, considerando-se as duas estratégias do modo de potência constante, são mostrados nas Figuras 17, 18 e 19. Para obtenção desses figuras foi utilizado um nível de compensação série de 46,24\% e um valor de 0,25 p.u. de potência ativa como referência do controlador PI. Observa-se, analisando as Figuras 17 e 19, que o torque de amortecimento é sempre maior para a estratégia de controle associada a maior tensão do SSSC. Já na Figura 18 é verificado que, para carregamentos inferiores a 0,5 p.u., quanto maior a tensão do SSSC, maior é o torque sincronizante. Por outro lado, para carregamentos superiores, o torque sincronizante resultante da aplicação das duas estratégias de controle é bastante próximo. Isto pode ser explicado considerando a contribuição do regulador automático de tensão do gerador no torque sincronizante. Para valores elevados de carregamento o regulador automático de tensão contribui para o aumento do torque sincronizante (Kundur, 1994). Então, nesse caso, sua contribuição no torque sincronizante resultante é muito maior do que a contribuição do SSSC. Assim, conclui-se que o SSSC operando no modo de potência constante tem influência bastante limitada no torque sincronizante do sistema, independentemente da estratégia de controle adotada. 

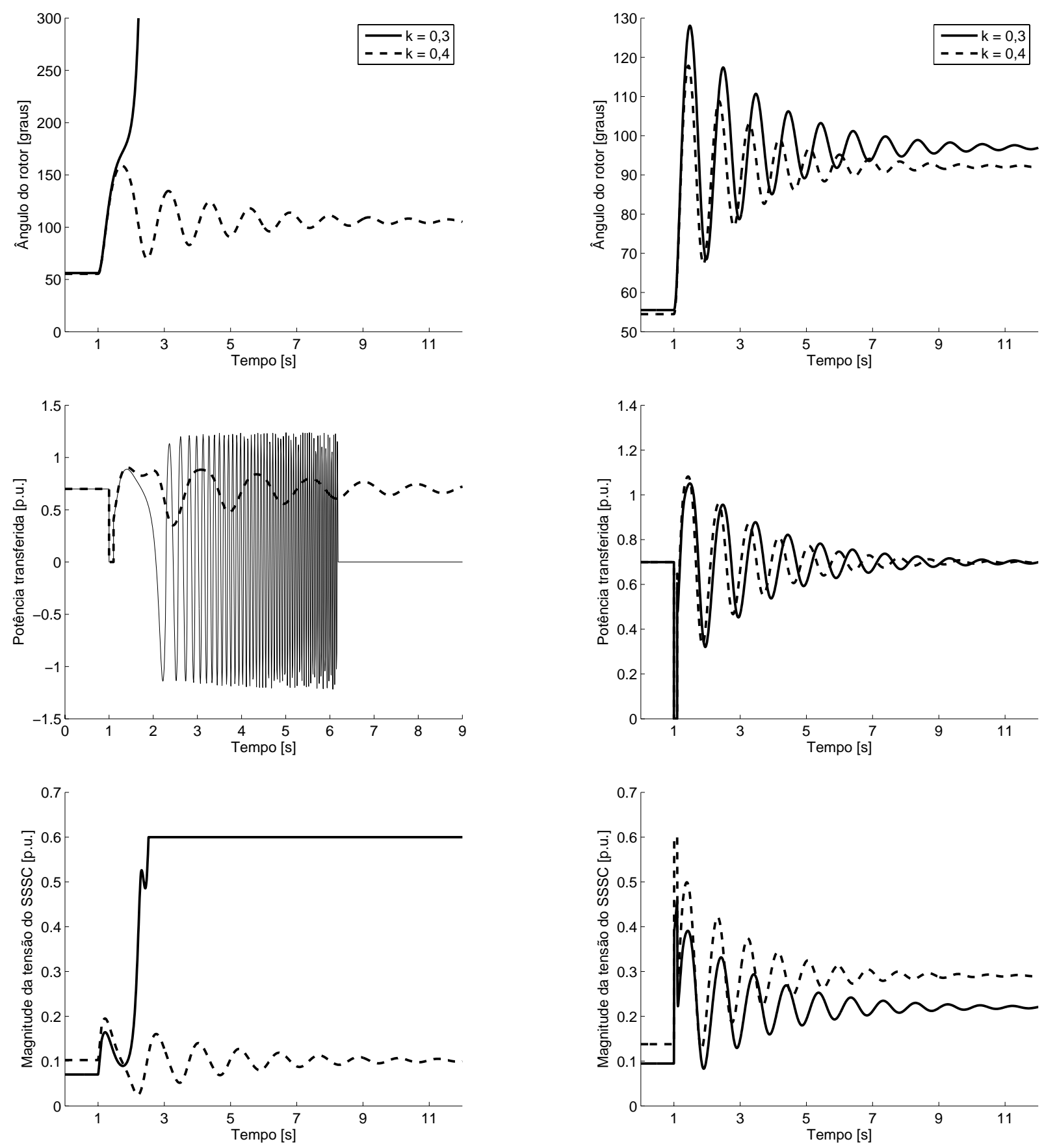

Figura 20: Resposta do sistema ao curto circuito trifásico com o SSSC no modo de tensão constante.

Figura 21: Resposta do sistema ao curto circuito trifásico com o SSSC no modo de reatância constante.

\section{ANÁLISE DA ESTABILIDADE TRANSI- TÓRIA}

Para investigar a influência do modo de operação do SSSC na estabilidade transitória do sistema teste da Figura 6 foram realizadas simulações considerando a ocorrência de um

curto circuito trifásico na linha 2-4 em um ponto próximo à barra $2 \mathrm{em} \mathrm{t}=1 \mathrm{~s}$ eliminado após $100 \mathrm{~ms}$ por meio da desconexão da linha 2-4. Esta é a falta simulada para obtenção de todos resultados apresentados nesta seção. Na Figura 20 é mostrada a resposta do sistema quando o SSSC opera no modo de tensão constante e a potência ativa transferida do 
gerador ao barramento infinito é de 0,7 p.u.. Dois níveis de compensação série da linha 2-3-4 são considerados; $30 \%$ e $40 \%$. A figura está dividida em três partes e as variáveis apresentadas são, respectivamente, o ângulo do rotor, a potência transferida e a magnitude da tensão do SSSC. Verifica-se que, para $\mathrm{k}=0,4 \mathrm{o}$ gerador permanece em sincronismo, o que não ocorre para $\mathrm{k}=0,3$. É observado que, no caso estável, as oscilações subsequentes à eliminação do defeito são adequadamente amortecidas pela ação da malha de controle de estabilidade e também que o valor da tensão $V_{s}$ é a mesmo nos pontos de operação pré-perturbação e pós-perturbação. Por outro lado, no caso instável, o crescimento monotônico do ângulo do rotor provoca também um crescimento indeterminado da tensão $V_{s}$ até que seu limite máximo seja alcançado.

A resposta do sistema para o SSSC operando no modo de reatância constante é apresentada na Figura 21. Observa-se que, neste caso, tanto para $\mathrm{k}=0,3$ quanto para $\mathrm{k}=0,4$ o gerador permanece em sincronismo. Verifica-se também que o valor da tensão do SSSC no ponto de operação pós-perturbação é maior do que no ponto pré-perturbação. Tal aumento é causado pelo acréscimo de magnitude da corrente que passa pela linha compensada. Na Figura 22, apresenta-se uma comparação entre o modo de tensão constante com $\mathrm{k}=0,5$ e o modo de reatância constante com $\mathrm{k}=0,3$ e 0,5, quando a potência transferida é 0,8 p.u.. É observado que o gerador permanece estável para os casos nos quais o SSSC opera no modo de reatância constante, mas não quando o mesmo opera com tensão constante, mesmo estando este último modo com um maior nível de compensação.

Considerando-se os modos de tensão e reatância constante, simulações foram realizadas para obtenção da potência crítica do sistema, ou seja, a máxima potência que pode ser transferida ao barramento infinito sem que o gerador perca a estabilidade devido a ocorrência de uma falta. A potência crítica é obtida por meio de repetidas simulações com pequenos incrementos na potência gerada (1\%). A falta aplicada para obtenção dos resultados é a mesma descrita anteriormente. Na Figura 23 é apresentada a potência crítica do sistema em função do nível de compensação série. É observado que, para um mesmo nível de compensação, a potência crítica é sempre maior quando o SSSC opera no modo de reatância constante. Observa-se também que a operação do SSSC no modo de reatância constante com um nível de compensação de $20 \%$ fornece maior valor de potência crítica do que a operação com tensão constante e nível de compensação de 50\%. Na Figura 24 é mostrado o ganho de potência crítica em relação ao caso do sistema sem compensação série na linha 2-3-4. É verificado que para um nível de compensação de $50 \%$ o modo de tensão constante propicia um ganho em torno de $16 \%$, enquanto que, usando esse mesmo nível de compensação, o ganho alcançado com o modo de reatância constante é de cerca de $43 \%$.
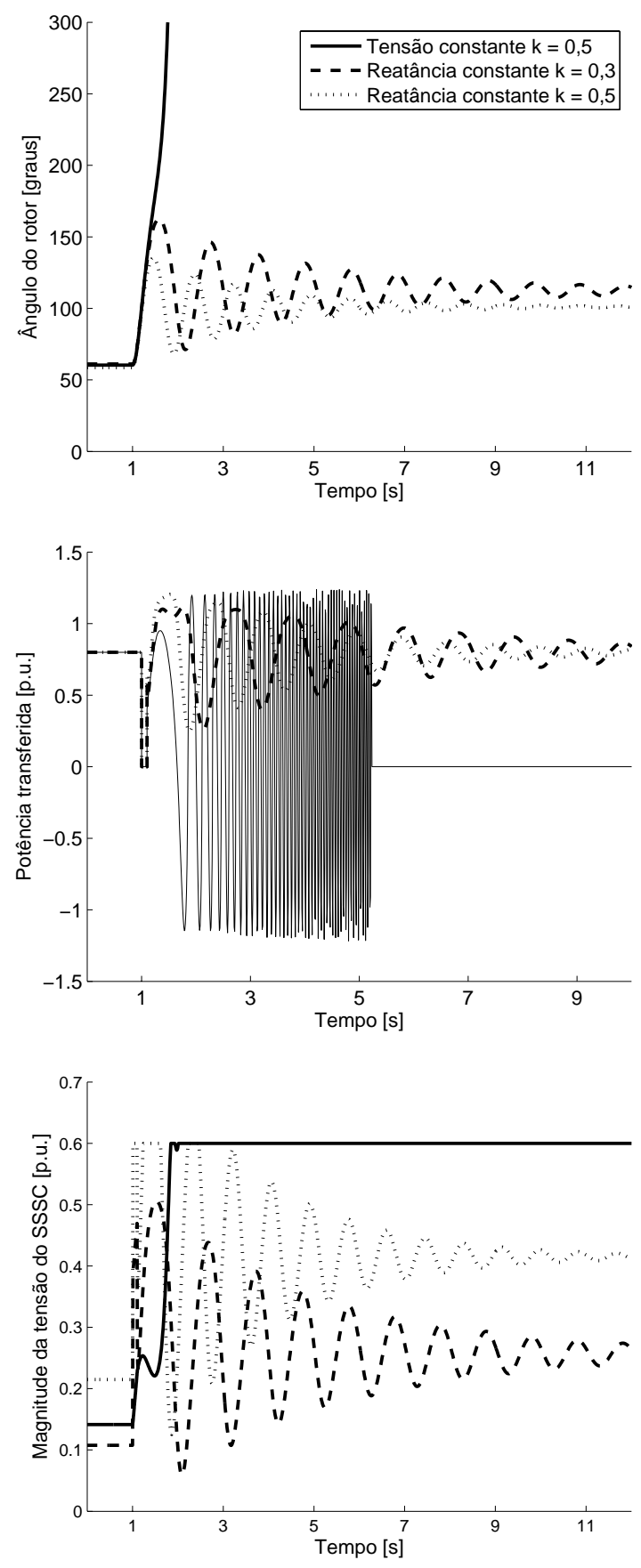

Figura 22: Resposta do sistema ao curto circuito trifásico com o SSSC nos modos de tensão e reatância constante.

O motivo pelo qual o SSSC no modo de reatância constante exibe um desempenho bastante superior do que no modo de tensão constante para o aumento do limite de estabilidade transitória pode ser melhor entendido por meio do critério da igualdade de áreas. Com esse propósito, considere o sistema radial mostrado na Figura 25, o qual compreende um gera- 


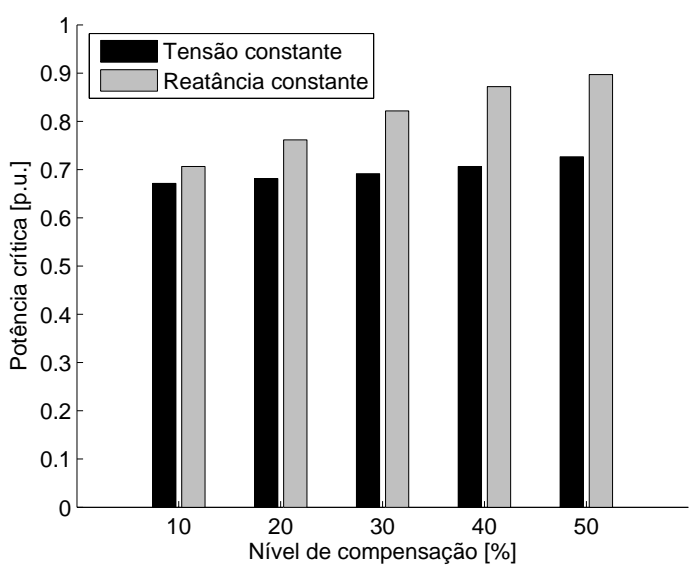

Figura 23: Potência crítica em função do nível de compensação.

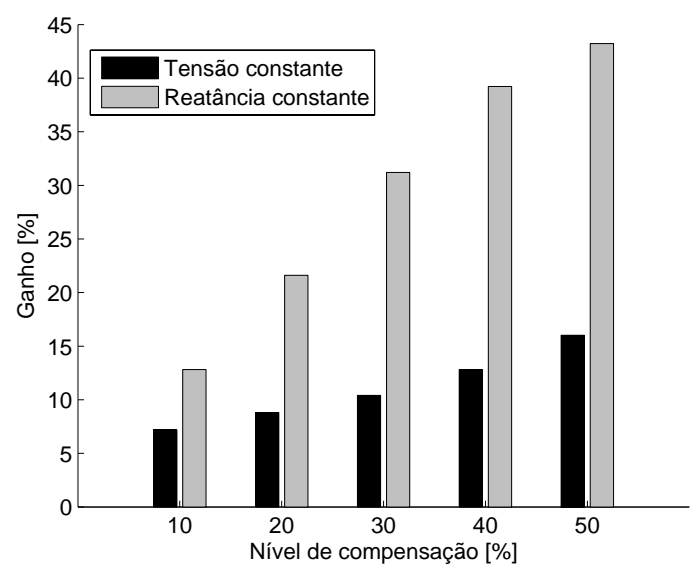

Figura 24: Ganho na potência crítica em função do nível de compensação.

dor conectado ao barramento infinito por meio de uma linha de transmissão compensada por um SSSC. Nas Figuras 26 e 27 são mostradas as curvas potência-ângulo do gerador para o SSSC operando no modo de tensão e reatância constante, respectivamente, desconsiderando-se o impacto do regulador automático de tensão do gerador. Essas curvas foram obtidas para um nível de compensação série de $50 \%$ associado ao carregamento de 0,5 p.u.. Também é mostrada a curva potência-ângulo do gerador para o sistema sem compensação série $(k=0)$. Observa-se que os dois modos de operação do SSSC aumentam a capacidade de transferência de potência do gerador ao barramento infinito. Entretanto, a potência máxima que pode ser transferida é bem maior quando o SSSC opera no modo de reatância constante. Enquanto o modo de tensão constante permite um aumento em torno de $18 \%$, o modo de reatância constante permite um aumento de
$100 \%$ na capacidade de transferência de potência do sistema.

Considera-se a ocorrência de uma falta trifásica na barra terminal do gerador quando o mesmo entrega 0,5 p.u. de potência ativa ao barramento infinito. Na Figura 26, $\delta_{0}$ e $\delta_{c l}$ representam os ângulos nos instantes nos quais a falta é iniciada e eliminada, respectivamente. O ângulo máximo que o gerador pode atingir sem que ocorra perda de estabilidade é representado por $\delta_{m}$. Estas mesmas definições são adotadas para os ângulos $\delta_{0}^{\prime}, \delta_{c l}^{\prime}$ e $\delta_{m}^{\prime}$ da Figura 27. As áreas $A_{1}$ e $A_{1}^{\prime}$ representam a energia cinética acumulada pelo sistema rotativo do gerador durante a falta, e as áreas $A_{2}$ e $A_{2}^{\prime}$ representam a energia liberada após a eliminação do defeito. O critério da igualdade de áreas estabelece que, se $A_{1} \leq A_{2}$ o sistema é estável, e se $A_{2} \leq A_{1}$ o sistema é instável (Kundur, 1994). Comparando as Figuras 26 e 27, observa-se que, enquanto $A_{1}=A_{1}^{\prime}, A_{2}$ é bem menor que $A_{2}^{\prime}$, indicando que o SSSC operando no modo de reatância constante fornece um maior limite de estabilidade transitória ao sistema.

O uso de um SSSC para controlar o fluxo de potência na rede é somente possível em sistemas nos quais existam duas ou mais linhas de transmissão em paralelo. Considerando o sistema teste da Figura 6, a ação do controlador PI deve ser obstruída durante desconexões da linha 2-4, caso contrário sérios problemas de controle podem surgir visto que tal controlador tentaria realizar uma função impossível de ser realizada. Assim, uma lógica de proteção que iniba a ação do controlador PI durante contingências críticas deve ser adotada. Durante tais contingências, uma opção é usar uma lógica que mude o modo de operação do SSSC para o modo de reatância constante, uma vez que tal modo é o que melhor contribui para a melhora da estabilidade transitória do sistema, conforme mostrado nesta seção.

Em casos nos quais faltas são eliminadas sem mudança na configuração do sistema, ou seja, sem perda de linhas, as respostas do sistema com o SSSC operando no modo de potência constante ou no modo de tensão constante são muito similares. Isto ocorre porque o controlador PI é de ação lenta e, assim, as variações da tensão $V_{s}$ serão muito pequenas no intervalo de tempo de interesse, por volta de até $3 \mathrm{~s}$ após eliminação da falta. Tal fato foi confirmado em simulações (não mostradas no trabalho) nas quais a mesma falta trifásica aplicada anteriormente foi eliminada sem o desligamento da linha 2-4.

\section{CONCLUSÕES}

Diferentes modos de operação do SSSC podem causar diferentes impactos na estabilidade angular de um sistema de potência. Um SSSC pode ser usado para realizar puramente compensação série de potência reativa ou para controlar o fluxo de potência na rede de transmissão. Um estabilizador 


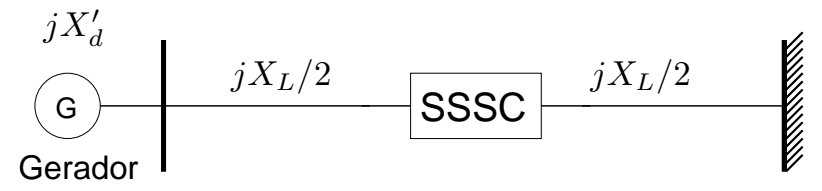

Figura 25: Sistema radial com SSSC.

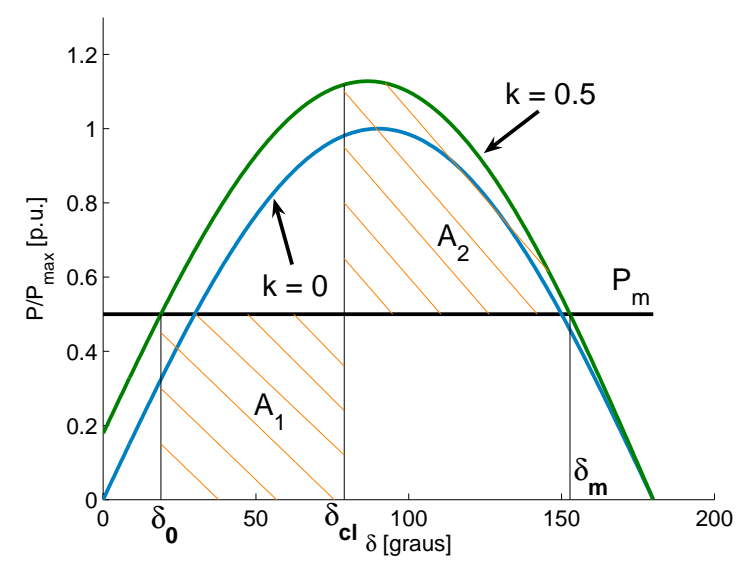

Figura 26: Curva potência-ângulo do gerador com o SSSC operando no modo de tensão constante.

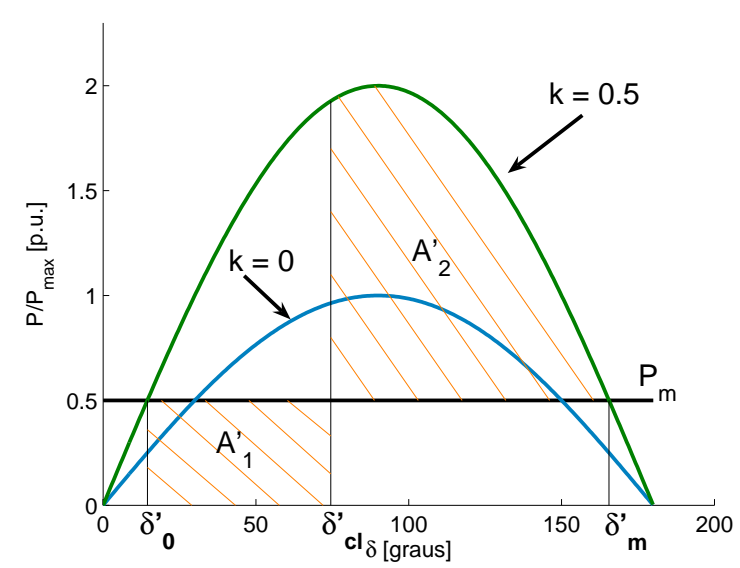

Figura 27: Curva potência-ângulo do gerador com o SSSC operando no modo de reatância constante.

também pode ser incluído em seu sistema de controle para fornecer torque de amortecimento ao sistema. Neste trabalho foi realizada uma investigação da influência do modo de operação do SSSC na estabilidade de ângulo a pequenas perturbações e na estabilidade transitória. Além disso, é feita uma comparação entre o fluxo de potência ativa e a magnitude da corrente como sinais candidatos para a realimentação do con- trolador POD. Todas simulações apresentadas foram obtidas usando o programa PSAT. Os resultados obtidos permitem concluir que operar o SSSC no modo de reatância constante é a melhor estratégia para estender os limites de estabilidade a pequenas perturbações e de estabilidade transitória. Também é destacado que a utilização da magnitude da corrente como sinal de entrada do controlador POD resulta em um melhor desempenho dinâmico do sistema.

\section{AGRADECIMENTOS}

Os autores são gratos ao $\mathrm{CNPq}$ e à FAPESP pelo suporte financeiro fornecido a esta pesquisa.

\section{REFERÊNCIAS}

Del Rosso, A. D., Cañizares, C. A. e Doña, V. M. (2003). A study of TCSC controller design for power system stability improvement, IEEE Transactions on Power Systems 18(4): 1487-1496.

Duangkamol, K., Mitani, Y., Tsuji, K. e Hojo, M. (2000). Fault current limiting and power system stabilization by static synchronous series compensator, IEEE Power System Technology, Proceedings. PowerCon 2000 3(8): 1581-1586.

Elices, A., Rouco, L., Bourles, H. e Margotin, T. (2004). Physical interpretation of state feedback controllers to damp power system oscillations, IEEE Transactions on Power Systems 19(1): 436-443.

Gama, C. (1999). Brazilian north-south interconnection - control application and operating experience with a TCSC, IEEE PES Summer Meeting 2: 1103-1108.

Hingorani, N. G. e Gyugyi, L. (2000). Concepts and Technology of Flexible AC Transmission Systems, IEEE Press - Jon Wiley \& Sons.

Kumkratug, P. e Haque, M. H. (2003). Improvement of stability region and damping of a power system by using SSSC, IEEE PES .

Kundur, P. (1994). Power System Control and Stability, Editora Mc Graw-Hill.

Martins, N. e Lima, L. (1990). Eigenvalue and frequency domain fo small-signal electromechanical stability problems, IEEE Symposium on Application of Eigenanalysis and Frequency Domain Methods for System Dynamic Performance Special Publication 90TH0292-3 PWR: 17-33.

Martins, N., Pinto, H. J. C. P. e Paserba, J. J. (1999). TCSC controls for line scheduling and system oscillation dam- 
ping - results for a small example system, Proceedings of 13th Power System Computation Conference (PSCC), Trondheim, Norway pp. 1244-1251.

Mehraban, A., Edris, A., Schauder, C. e Provanzana, J. H. (1998). Installation, commissioning, and operation of the world's firt UPFC on the AEP system, Proceedings of International Conference on Power System Technology (POWERCON) 1: 323-327.

Milano, F. (2005). An open source power system analysis toolbox, IEEE Transactions on Power Systems 20(3): 1199-1206.

Milano, F. (2006). Power System Analysis Toolbox - Documentation for PSAT version 2.0.0 $\beta$. http: //www. power. uwaterloo.ca/ $\sim$ fmilano/downloads.htm.

Song, Y. H. e Johns, A. T. (1999). Flexible AC Transmission System (FACTS), The Institute of Electrical Engineers.

Watanabe, E. H., Barbosa, P. G., Almeida, K. C. e Taranto, G. N. (1998). Tecnologia FACTS - tutorial, SBA Controle \& Automação 9(1): 39-55.

Zhang, X.-P. (2003). Advanced modeling of the multicontrol functional static synchronous series compensator (SSSC) in newton power flow, IEEE Transactions on Power Systems 18(4): 1410-1416.

\section{APÊNDICE}

Dados do gerador em p.u. (Kundur, 1994)

$$
\begin{array}{lcl}
R_{a}=0.003 & X_{L}=0.15 & X_{d}=1.81 \\
X_{d}^{\prime}=0.30 & X_{d}^{\prime \prime}=0.23 & T_{d o}^{\prime}=8.0 \mathrm{~s} \\
T_{d o}^{\prime \prime}=0.03 \mathrm{~s} & X_{q}=1.76 & X_{q}^{\prime}=0.65 \\
X_{q}^{\prime \prime}=0.25 & T_{q o}^{\prime}=1.0 \mathrm{~s} & T^{\prime \prime} q o=0.07 \mathrm{~s} \\
H=3.5 & D=0.0 &
\end{array}
$$

Dados do regulador de tensão em p.u. (Kundur, 1994)

$K_{a}=200 \quad T_{1}=1.0 \mathrm{~s} \quad T_{2}=1.0 \mathrm{~s}$

$T_{r}=0.015 \mathrm{~s} \quad V r_{\max }=7.0 \quad V r_{\min }=-6.4$

Dados do transformador e do circuito de transmissão em p.u. (Kundur, 1994)

$X_{12}=0.15 \quad X_{24}=0.5 \quad X_{23}+X_{34}=0.93$ 